\title{
Associations between maternal lipid profile and pregnancy complications and perinatal outcomes: a population-based study from China
}

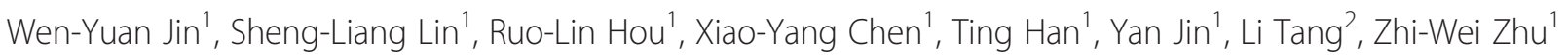
and Zheng-Yan Zhao ${ }^{1 *}$

\begin{abstract}
Background: Dyslipidemia in pregnancy are associated with gestational diabetes mellitus (GDM), preeclampsia, preterm birth and other adverse outcomes, which has been extensively studied in western countries. However, similar studies have rarely been conducted in Asian countries. Our study was aimed at investigating the associations between maternal dyslipidemia and adverse pregnancy outcomes among Chinese population.

Methods: Data were derived from 934 pairs of non-diabetic mothers and neonates between 2010 and 2011 . Serum blood samples were assayed for fasting total cholesterol (TC), triglycerides (TG), high-density lipoprotein-cholesterol (HDL-C), and low-density lipoprotein-cholesterol (LDL-C) concentrations during the first, second and third trimesters. The present study explored the associations between maternal lipid profile and pregnancy complications and perinatal outcomes. The pregnancy complications included GDM, preeclampsia and intrahepatic cholestasis of pregnancy (ICP); the perinatal outcomes included preterm birth, small/large for gestational age (SGA/LGA) infants and macrosomia. Odds ratios (ORs) and $95 \%$ confidence intervals (95\% Cls) were calculated and adjusted via stepwise logistic regression analysis. Optimal cut-off points were determined by ROC curve analysis.

Results: After adjustments for confounders, every unit elevation in third-trimester TG concentration was associated with increased risk for $\mathrm{GDM}(\mathrm{OR}=1.37,95 \% \mathrm{Cl}: 1.18-1.58)$, preeclampsia $(\mathrm{OR}=1.50,95 \% \mathrm{Cl}: 1.16-1.93)$, ICP $(\mathrm{OR}=1.28,95 \% \mathrm{Cl}: 1.09-1.51), \mathrm{LGA}(\mathrm{OR}=1.13,95 \% \mathrm{Cl}: 1.02-1.26)$, macrosomia (OR=1.19, $95 \% \mathrm{Cl}: 1.02-1.39)$ and decreased risk for SGA (OR $=0.63,95 \% \mathrm{Cl}$ : 0.40-0.99); every unit increase in HDL-C concentration was associated with decreased risk for GDM and macrosomia, especially during the second trimester (GDM: OR=0.10, $95 \%$ Cl: 0.03-0.31; macrosomia: OR = 0.25, $95 \%$ Cl: 0.09-0.73). The optimal cut-off points for third-trimester TG predicting GDM, preeclampsia, ICP, LGA and SGA were separately $\geq 3.871,3.528,3.177,3.534$ and $\leq 2.530 \mathrm{mmol} / \mathrm{L}$. The optimal cut-off points for third-trimester HDL-C identifying GDM, macrosomia and SGA were respectively $\leq 1.712,1.817$ and $\geq 2.238 \mathrm{mmol} / \mathrm{L}$.

Conclusions: Among Chinese population, maternal high TG in late pregnancy was independently associated with increased risk of GDM, preeclampsia, ICP, LGA, macrosomia and decreased risk of SGA. Relative low maternal $\mathrm{HDL}-\mathrm{C}$ during pregnancy was significantly associated with increased risk of GDM and macrosomia; whereas relative high HDL-C was a protective factor for both of them.
\end{abstract}

\footnotetext{
* Correspondence: zhaozy@zju.edu.cn

'Department of Children's Health Care, Children's Hospital, Zhejiang

University School of Medicine, Hangzhou 310003, China

Full list of author information is available at the end of the article
} 


\section{Background}

Evidence has suggested that adverse pregnancy outcomes can jeopardize short- and long-term maternal and fetal health. Women with previous gestational diabetes mellitus (GDM) have an increased risk of type 2 diabetes or cardiovascular diseases (CVD) in later life [1, 2]. Pregnancies accompanied with preeclampsia have the predisposition to future CVD and metabolic syndrome [3-5]. According to Developmental origins of Health and Disease (DOHaD) hypothesis, individuals who were exposed to an undernourished intrauterine environment are more prone to developing type 2 diabetes or CVD in later life as a result of epigenetic modifications, which permanently reprogramme their bodies [6, 7]. Therefore, it is of great importance to explore effective interventions to prevent these adverse outcomes. It is noteworthy that disturbed maternal metabolism, including atherogenic lipid changes in pregnancy, is one of the crucial factors that involved in pathological process.

From the $12^{\text {th }}$ week of pregnancy, lipid parameters, including total cholesterol (TC), triglycerides (TG), low-density lipoprotein-cholesterol (LDL-C), highdensity lipoprotein-cholesterol (HDL-C) and phospholipid gradually increase, especially in the second and third trimesters [8-13]. Previous research has indicated that pregnancy-induced hyperlipidemia contributes to an increased morbidity of GDM and preeclampsia. At all gestational stages, high TG concentrations were associated with a raised risk for gestational impaired glucose tolerance (GIGT) and GDM [14-16]. Results of a few studies have suggested a concentration-dependent positive association between maternal TG and the risk for preeclampsia [17-20]. Moreover, evidence has shown pregnant women with higher oxidized LDL-C concentrations are also at greater risk of developing preeclampsia [21-23]. In addition, Amsterdam Born Children and Their Development cohort study discovered that maternal TG concentrations in early pregnancy were linearly related with the prevalence of pregnancy-induced hypertension, preeclampsia, induced preterm birth (PTB) and large for gestational age (LGA) [24].

Except for pregnancy complications, maternal dyslipidemia is also closely linked with adverse perinatal outcomes. Catov et al. [25] reported that both elevated cholesterol and TG in early pregnancy were associated with an increased risk of spontaneous PTB. Furthermore, maternal impaired TG and non-esterified fatty acids (NEFA) metabolism were correlated with excessive fetal growth [26, 27]. In women diagnosed with GDM, it has been demonstrated that maternal TG and NEFA concentrations in late pregnancy are positively correlated with newborns' birth weight, body mass index (BMI) and fat mass [28-30]. Interestingly, other clinical trials have indicated maternal fasting TG is positively correlated with neonatal body weight even in women with normal glucose tolerance but positive diabetic screenings [31, 32]. Additionally, recent studies have described a positive association between maternal TG concentrations and the risk for LGA newborns independent of glycemic control [24, 31, 33].

Despite these findings, there are still some controversies on the relationship between maternal lipid disturbances and certain pregnancy complications or perinatal outcomes. For instance, GDM was discovered to be associated with lower maternal LDL-C and HDL-C concentrations during the second and third trimesters in several studies $[15,34-36]$. Nevertheless, other assays showed no significant difference in the concentrations of LDL-C and HDL-C between GDM and normal pregnancies [34, 36-38]. Additionally, as mentioned before, there exists a debate focused on whether maternal TG is positively correlated with neonatal birth weight only in GDM/ diabetic subjects or also in nondiabetic ones [28-33, 39]. These conflicting results could potentially be explained by differences in trimester of pregnancy, condition of glycemic control, race/ethnicity and sample size, but the real causes remain unknown.

Our study was aimed at comprehensively investigating the associations between Chinese maternal lipid profile and adverse pregnancy outcomes, i.e. GDM, preeclampsia, intrahepatic cholestasis of pregnancy (ICP), PTB, LGA, small for gestational age (SGA) and macrosomia. At the same time, we attempted to advance understandings and provide some potent evidence for elucidating the existing controversies.

\section{Methods}

\section{Study population}

Between 30 June 2010 and 30 June 2011, pregnant women who attended regular prenatal health care and intended to give a birth in Women's Hospital, Zhejiang University School of Medicine were invited to participate in the study. Before enrollment, our study was approved by the hospital's Clinical Research Ethics Committee and written informed consent was signed by every participant. We established the study cohort based on inclusion and exclusion criteria. Inclusion criteria of pregnant women were: 1) pregnant at 28-37 gestational weeks; 2) had integrated medical records and clear gestational age; 3) singleton pregnancy; and 4) naturally conceived. Exclusion criteria of pregnant women were: 1) multiple pregnancy; 2) had diabetes mellitus, chromosomal abnormalities, inherited metabolic diseases or thyroid diseases before pregnancy; 3) experienced serious infection during early pregnancy; and 4) conceived with assisted reproductive techniques. A total of 1020 expectant mothers were recruited. All the women included were requested to complete an extensive questionnaire about maternal age, 
height, parity, prepregnancy weight, gestational weight gain (GWG), lifestyle, education background, family income and other important information. Data on firsttrimester and second-trimester lipid concentrations, GDM, preeclampsia and ICP were collected from medical records. They were followed from recruitment to delivery. Information on delivery mode, gestational age, newborn sex, birth weight, Apgar scores and perinatal outcomes were recorded by midwives or obstetricians upon delivery. Inclusion criteria for newborns were singleton and 5-min-postpartum Apgar scores $\geq 7$. Exclusion criteria for newborns were chromosomal abnormalities, inherited metabolic diseases and congenital abnormalities. Finally, 934 pairs of mothers and neonates were eligible for our study.

\section{Biochemical analyses}

Venous blood samples were taken after overnight fasting from all the participants at the first (7-10 gestational weeks), second (21-24 gestational weeks) and third (33-37 gestational weeks) trimester of pregnancy. The blood samples were collected in a $3.5-\mathrm{ml}$ vacutainer (BD Plymouth, PL6 7BP, UK, SST II Advance Tubes) for the preparation of serum. At the biochemical laboratory of Women's Hospital, Zhejiang University School of Medicine, 1-ml aliquots of serum samples were obtained by centrifugation (3500 rpm for $10 \mathrm{~min}$ at $4{ }^{\circ} \mathrm{C}$ ) and stored at $-20{ }^{\circ} \mathrm{C}$ until analysis. Every sample was assayed for TC, TG, HDL-C and LDL-C concentrations. TC was assayed with the cholesterol oxidase-phenol aminophenazone method and TG was assayed using the glycerol-3-phosphatase oxidasephenol aminophenazone method. HDL-C and LDL-C were measured by homogeneous enzymatic colorimetric assays. All the lipid measurements were performed on an automatic biochemical analyser (Abbott Architect C16000, Abbott Laboratories, USA) respectively with TC, TG, HDL-C and LDL-C detection kits (Abbott Diagnostic Kit, Abbott Laboratories, USA). The interand intra-assay coefficients of variation were $<1.9 \%$, $0.81 \%$ (TC); $<1.9 \%, 1.6 \%$ (TG); $<1.0 \%, 0.44 \%$ (HDLC) and $<1.9 \%, 1.5 \%$ (LDL-C), respectively.

\section{Definitions}

BMI was calculated by dividing weight in kilograms by the square of height in meters. Maternal prepregnancy BMI was categorized into underweight $\left(<18.5 \mathrm{~kg} / \mathrm{m}^{2}\right)$, normal weight $\left(18.5-24.9 \mathrm{~kg} / \mathrm{m}^{2}\right)$, overweight $\left(25.0-29.9 \mathrm{~kg} / \mathrm{m}^{2}\right)$, and obese $\left(\geq 30.0 \mathrm{~kg} / \mathrm{m}^{2}\right)$ groups on the basis of World Health Organization BMI classification [40]. According to the new recommendations from American Institute of Medicine, GWG was stratified into appropriate, inadequate and excessive groups [41]. Based on different prepregnancy BMIs, appropriate GWG was defined as
$12.5-18.0 \mathrm{~kg}$ in underweight women, $11.5-16.0 \mathrm{~kg}$ in normal weight women, $7.0-11.5 \mathrm{~kg}$ in overweight women and $5.0-9.0 \mathrm{~kg}$ in obese women. Exceeding the thresholds was defined as excessive GWG, while falling below the thresholds was defined as inadequate GWG.

GDM was diagnosed according to the recommendations from International Association of Diabetes and Pregnancy Study Groups [42, 43]. The proposed criteria for GDM were as follows: Before an oral glucose tolerance test (OGTT), every participant was requested for a $50 \mathrm{~g}$ glucose challenge test and serum glucose levels were assayed $1 \mathrm{hr}$ later. Subjects with positive results (glucose levels $\geq 7.8 \mathrm{mmol} / \mathrm{L}$ ) were required to undergo a $75 \mathrm{~g}$ OGTT. Serum glucose levels during OGTT were measured at 0,1 and $2 \mathrm{hr}$, respectively. The normal values were fasting glucose $<5.1 \mathrm{mmol} / \mathrm{L}, 1-\mathrm{hr}$ glucose $<10.0 \mathrm{mmol} / \mathrm{L}$ and $2-\mathrm{hr}$ glucose $<8.5 \mathrm{mmol} / \mathrm{L}$. If one or more values equaled or exceeded the above thresholds, women were diagnosed as having GDM.

Hypertensive disorders in pregnancy were a set of diseases including pregnancy-induced hypertension, preeclampsia, eclampsia, chronic hypertension complicating pregnancy and preeclampsia superimposed upon chronic hypertension. Preeclampsia was defined as a specific pregnancy-induced disorder characterized with hypertension (systolic blood pressure $\geq 140 \mathrm{mmHg}$ and/or diastolic blood pressure $\geq 90 \mathrm{mmHg}$ ) and significant proteinuria (urine protein $\geq 300 \mathrm{mg} / 24 \mathrm{~h}$ or positive results in random urine protein tests) in previously normotensive women on or after 20 weeks gestational age [44].

ICP is a pregnancy-specific disorder typically occurs in the third trimester characterized by pruritus and jaundice. Confirmation of diagnosis relied on abnormal liver function tests and raised maternal serum bile acids [45]. Abnormal liver function tests included elevated levels of alanine aminotransferase, aspartate aminotransferase and/or gamma-glutamyl transpeptidase. The upper limits of total serum bile acids were 10-14 micromoles/ $\mathrm{L}$ in postprandial state and $6-10$ micromoles/L in fasting state. Exceeding the upper limits was an important diagnostic basis of ICP.

Preterm birth was defined as birth of newborns less than 37 weeks gestational age. Newborns were classified into appropriate for gestational age (AGA), SGA and LGA on the basis of Neonatal Birth Weight for Gestational Age and Percentile in 15 Cities of China [46]. Neonates were defined as SGA if their birth weights fell below the $10^{\text {th }}$ percentile for gestational age and as LGA if their birth weights exceeded the $90^{\text {th }}$ percentile for gestational age. AGA was defined as birth weights met or exceeded the $10^{\text {th }}$ percentile and met or fell below the $90^{\text {th }}$ percentile for gestational age. According to the birth weight, neonates could be stratified into low birth weight $(<2500 \mathrm{~g})$, 
normal birth weight (2500-4000 g) and macrosomia (>4000 g) groups.

\section{Statistical analysis}

In our study, normally and non-normally distributed continuous variables were respectively presented as mean \pm standard deviation (SD) and median (interquartile range, IQR), categorical variables were presented as N (\%). Serum TC, TG, LDL-C, HDL-C concentrations at the first, second and third trimesters were compared by Kruskal-Wallis test. Maternal lipid increases from the first to second trimester and the second to third trimester were compared using Mann-Whitney test. Forward stepwise logistic regression analysis were applied to explore the associations between maternal dyslipidemia and pregnancy complications and perinatal outcomes. We considered GDM, preeclampsia and ICP as pregnancy complications; and PTB, SGA, LGA, macrosomia as perinatal outcomes. In the multivariable adjusted model, maternal age, prepregnancy BMI, GWG, parity, cigarette exposure, socioeconomic status, infant sex and delivery mode were regarded as confounding variables. ROC curve analysis was conducted to determine the optimal cut-off points of TG, HDL-C and LDL-C for predicting maternal and neonatal adverse outcomes. Each optimal cut-off point was assessed via searching for the maximum value of sensitivity + specificity-1 (Youden index). Area under curve (AUC) was calculated to evaluate the predictive powers. All the analyses were performed with SPSS version 19.0 for Windows (SPSS Inc, Chicago, III, USA). P values $<0.05$ were defined as statistically significant.

\section{Results}

\section{Maternal lipid profile by trimester}

Table 1 shows maternal lipid profile by trimester. Serum TC, TG, LDL-C, HDL-C concentrations increased as pregnancy advanced. All the lipid parameters of the third trimester were higher than those of the first and second trimesters $(p<0.001)$. Furthermore, the increases in lipid concentrations from the second to third trimester were larger than those from the first to second trimester $(p<0.001)$. From the second to third trimester,
TC, TG, LDL-C and HDL-C concentrations increased by $35.5 \%, 24.1 \%, 13.4 \%$ and $9.0 \%$, respectively. In contrast, the data was separately $12.9 \%$ (TC), $12.3 \%$ (TG), $10.2 \%$ (LDL-C), $4.8 \%$ (HDL-C) from the first to second trimester.

\section{Characteristics of study population}

Table 2 presents maternal and neonatal characteristics of our study population. Among the 934 eligible mothers in the present study, the mean (SD) age at delivery was 29.21 (3.76) years old. Approximately $83 \%$ of them were nulliparous and about $7 \%$ of them were stratified as overweight or obese with prepregnancy $\mathrm{BMI} \geq 25.0 \mathrm{~kg} / \mathrm{m}^{2}$. Their mean (SD) prepregnancy BMI was $20.66(2.70) \mathrm{kg} /$ $\mathrm{m}^{2}$. The prevalence of maternal comorbidities, including GDM, preeclampsia and ICP was respectively $7.6 \%, 1.5 \%$ and $6.3 \%$. The mean (SD) gestational weight gain was $15.27(4.00) \mathrm{kg}$. According to the IOM recommendations for gestational weight gain, $54.0 \%$ met, $15.4 \%$ fell below and $30.6 \%$ exceeded the criteria. The newborns in our study had a mean (SD) birth weight of 3396.41 (436.52) g. $71.3 \%$ of them were AGA, $2.4 \%$ were SGA and $26.3 \%$ were LGA. $1.3 \%$ of them belonged to low birth weight group and $8.0 \%$ belonged to macrosomia group. The mean (SD) gestational age at birth was 38.84 (1.22) weeks.

\section{Associations between maternal lipid profile and pregnancy complications and perinatal outcomes}

Table 3 displays the associations between maternal third-trimester lipid profile and pregnancy complications and perinatal outcomes. Of all the expectant mothers, $7.6 \%$ developed GDM, $1.5 \%$ preeclampsia and $6.3 \%$ ICP. We observed every $\mathrm{mmol} / \mathrm{L}$ elevation in maternal third-trimester TG concentration was associated with an increased risk of GDM $[p<0.001$, adjusted odds ratio $(\mathrm{AOR})=1.37,95 \%$ confidence interval $(\mathrm{CI}): 1.18-1.58]$, preeclampsia $(p=0.002$, AOR $=1.50,95 \%$ CI: $1.16-1.93)$ and ICP $(p=0.002, \mathrm{AOR}=1.28,95 \% \mathrm{CI}: 1.09-1.51)$. In addition, every unit increase in LDL-C reduced the risk of $\mathrm{GDM}(p<0.001, \mathrm{AOR}=0.52,95 \% \mathrm{CI}: 0.38-0.72)$. While on the other hand, there were no significant associations between TC concentrations and all the above pregnancy complications.

Table 1 Maternal lipid profile by trimester

\begin{tabular}{llllllll}
\hline Lipids & First & Second & Third & First-Second & Second-Third & $p^{*}$ & $p^{* *}$ \\
\hline TC & $3.95(3.66-4.60)$ & $4.65(4.22-5.10)$ & $6.27(5.52-7.03)$ & $0.51(0.21-0.87)$ & $1.65(1.04-2.29)$ & 0.000 & 0.000 \\
TG & $2.20(1.77-2.73)$ & $2.45(2.11-2.89)$ & $3.06(2.37-3.98)$ & $0.27(0.09-0.51)$ & $0.59(0.12-1.11)$ & 0.000 & 0.000 \\
LDL-C & $2.25(2.08-2.45)$ & $2.46(2.22-2.77)$ & $2.87(2.32-3.45)$ & $0.23(0.03-0.46)$ & $0.33(-0.09-0.84)$ & 0.000 & 0.000 \\
HDL-C & $1.66(1.45-1.77)$ & $1.67(1.47-1.79)$ & $1.80(1.57-2.04)$ & $0.08(-0.12-0.21)$ & $0.15(-0.09-0.32)$ & 0.000 & 0.000 \\
\hline
\end{tabular}

TC total cholesterol, TG triglycerides, LDL-C/HDL-C low-density/high density lipoprotein-cholesterol

Maternal lipid concentrations and increases were presented as median (IQR) $\mathrm{mmol} / \mathrm{L}$

${ }^{*} p$ values were derived from the comparisons among lipid concentrations of the first, second, and third trimesters

${ }^{* *} p$ value were derived from the comparisons between lipid increases from the first to second trimester and the second to third trimester 
Table 2 Maternal and neonatal characteristics

\begin{tabular}{|c|c|}
\hline Characteristics & Mean \pm SD or N (\%) \\
\hline \multicolumn{2}{|l|}{ Maternal characteristics } \\
\hline Maternal age at delivery (year) & $29.21 \pm 3.76$ \\
\hline $20-29$ & $569(60.9)$ \\
\hline $30-34$ & $285(30.5)$ \\
\hline$\geq 35$ & $80(8.6)$ \\
\hline Prepregnancy BMI (kg/m²) & $20.66 \pm 2.70$ \\
\hline Underweight $(<18.5)$ & $192(20.7)$ \\
\hline Normal weight (18.5-24.9) & $669(72.1)$ \\
\hline Overweight and obese $(\geq 25.0)$ & $67(7.2)$ \\
\hline Gestational weight gain & $15.27 \pm 4.00$ \\
\hline Appropriate & $501(54.0)$ \\
\hline Inadequate & $143(15.4)$ \\
\hline Excessive & $284(30.6)$ \\
\hline \multicolumn{2}{|l|}{ Maternal education } \\
\hline$\geq$ University & $591(63.3)$ \\
\hline Junior college & $296(31.7)$ \\
\hline High school & $36(3.9)$ \\
\hline$\leq$ Junior school & $8(0.9)$ \\
\hline Unknown & $3(0.3)$ \\
\hline \multicolumn{2}{|l|}{ Family income (yuan/year) } \\
\hline$>200,000$ & $33(3.5)$ \\
\hline $100,000-200,000$ & $756(80.9)$ \\
\hline $50,000-100,000$ & $118(12.6)$ \\
\hline$<50,000$ & $19(2.0)$ \\
\hline Unknown & $8(0.9)$ \\
\hline \multicolumn{2}{|l|}{ Parity } \\
\hline Nulliparous & $778(83.3)$ \\
\hline Multiparous & $156(16.7)$ \\
\hline Exposed to cigarette during pregnancy & $59(6.3)$ \\
\hline GDM & $71(7.6)$ \\
\hline Preeclampsia & $14(1.5)$ \\
\hline ICP & $59(6.3)$ \\
\hline \multicolumn{2}{|l|}{ Delivery mode } \\
\hline Vaginal delivery & $216(23.1)$ \\
\hline Cesarean section & $718(76.9)$ \\
\hline \multicolumn{2}{|l|}{ Neonatal characteristics } \\
\hline Male infant & $493(52.8)$ \\
\hline Gestational age (GA) at delivery & $38.84 \pm 1.22$ \\
\hline Preterm (GA < 37 weeks) & $27(2.9)$ \\
\hline Full term ( $37 \leq \mathrm{GA}<42$ weeks) & $907(97.1)$ \\
\hline Birth weight(g) & $3396.41 \pm 436.52$ \\
\hline$<2500$ & $12(1.3)$ \\
\hline $2500-4000$ & $847(90.7)$ \\
\hline$>4000$ & $75(8.0)$ \\
\hline
\end{tabular}

Table 2 Maternal and neonatal characteristics (Continued)

\begin{tabular}{ll}
\hline Weight for gestational age & \\
SGA & $22(2.4)$ \\
AGA & $666(71.3)$ \\
LGA & $246(26.3)$ \\
\hline
\end{tabular}

$B M I$ body mass index, GDM gestational diabetes mellitus, ICP intrahepatic cholestasis of pregnancy, SGA/AGA/LGA small/appropriate/large for gestational age

In our study, the incidence of PTB, SGA, LGA and macrosomia was $2.9 \%, 2.4 \%, 26.3 \%$ and $8.0 \%$, respectively. Table 3 showed there was no significant association between third-trimester TG and PTB. However, multivariate analysis discovered that every $\mathrm{mmol} / \mathrm{L}$ increase in third-trimester TG concentration was associated with an increased risk for LGA $(p=0.025, \mathrm{AOR}=1.13,95 \% \mathrm{CI}$ : $1.02-1.26)$ and macrosomia $(p=0.024, \quad \mathrm{AOR}=1.19$, $95 \%$ CI: 1.02-1.39), a decreased risk for SGA ( $p=$ 0.046, $\mathrm{AOR}=0.63,95 \% \mathrm{CI}: 0.40-0.99)$. In contrast, there were no significant associations between $\mathrm{TC}$ and LDL-C concentrations and all the above perinatal outcomes.

Table 4 reports the associations between maternal HDL-C concentrations and GDM, macrosomia and SGA at all gestational stages. Significant negative associations were observed between HDL-C and the risk for GDM (during the whole pregnancy) and macrosomia (during the second and third trimesters). The strongest inverse associations appeared during the second trimester. Every unit increase in second-trimester HDL-C concentration was associated with a decreased risk for GDM $(p<0.001, \mathrm{AOR}=0.10,95 \% \mathrm{CI}: 0.03-0.31)$ and macrosomia $(p=0.011, \mathrm{AOR}=0.25,95 \% \mathrm{CI}$ : 0.09-0.73). Additionally, women with higher HDL-C concentrations were at a greater risk of having SGA offsprings and the relationship reached statistical significance at the third trimester ( $p=0.026, \mathrm{AOR}=3.15,95 \% \mathrm{CI}: 1.15-8.65)$.

Table 5 presents the optimal cut-off points of maternal third-trimester lipids for predicting pregnancy complications and perinatal outcomes. The optimal cut-off points proposed by ROC curve analysis for third-trimester TG in predicting GDM, preeclampsia, ICP, LGA and SGA were separately $\geq 3.871,3.528,3.177,3.534$ and $\leq 2.530 \mathrm{mmol} / \mathrm{L}$. TG predicting preeclampsia owned the strongest predictive power with the largest AUC [0.736 (95 \% CI: 0.5950.876)]. The optimal cut-off points for third-trimester HDL-C in identifying GDM, macrosomia and SGA were respectively $\leq 1.712,1.817$ and $\geq 2.238 \mathrm{mmol} / \mathrm{L}$. HDL-C identifying GDM had the strongest predictive ability with the largest AUC [0.617 (95 \% CI: 0.548-0.686)]. Besides, the optimal cut-off point for third-trimester LDL-C in predicting GDM was $\leq 2.415 \mathrm{mmol} / \mathrm{L}$.

Except for the last trimester, per unit elevation in TG concentrations could led to 1.58-fold increased risk of 
Table 3 Associations between maternal third-trimester lipid profile and pregnancy complications and perinatal outcomes

\begin{tabular}{|c|c|c|c|c|c|c|c|c|}
\hline \multirow[b]{2}{*}{ Outcomes } & \multicolumn{2}{|l|}{$\mathrm{TC}$} & \multicolumn{2}{|l|}{$\mathrm{TG}$} & \multicolumn{2}{|l|}{ LDL-C } & \multicolumn{2}{|l|}{$\mathrm{HDL}-\mathrm{C}$} \\
\hline & AOR $(95 \% \mathrm{Cl})$ & $p$ value & AOR $(95 \% \mathrm{Cl})$ & $p$ value & AOR $(95 \% \mathrm{Cl})$ & $p$ value & AOR $(95 \% \mathrm{Cl})$ & $p$ value \\
\hline GDM & $0.84(0.68-1.04)$ & 0.109 & $1.37(1.18-1.58)$ & 0.000 & $0.52(0.38-0.72)$ & 0.000 & $0.27(0.13-0.58)$ & 0.001 \\
\hline Preeclampsia & $0.99(0.64-1.54)$ & 0.979 & $1.50(1.16-1.93)$ & 0.002 & $0.65(0.33-1.31)$ & 0.228 & $0.48(0.10-2.26)$ & 0.352 \\
\hline ICP & $1.20(0.97-1.49)$ & 0.096 & $1.28(1.09-1.51)$ & 0.002 & $1.29(0.94-1.76)$ & 0.113 & $0.56(0.25-1.22)$ & 0.141 \\
\hline PTB & $0.80(0.57-1.11)$ & 0.180 & $1.04(0.77-1.38)$ & 0.818 & $0.83(0.52-1.32)$ & 0.430 & $1.05(0.37-3.00)$ & 0.931 \\
\hline SGA & $1.12(0.80-1.56)$ & 0.520 & $0.63(0.40-0.99)$ & 0.046 & $1.16(0.71-1.89)$ & 0.565 & $3.15(1.15-8.65)$ & 0.026 \\
\hline LGA & $0.98(0.86-1.11)$ & 0.715 & $1.13(1.02-1.26)$ & 0.025 & $0.93(0.78-1.11)$ & 0.418 & $0.79(0.52-1.21)$ & 0.281 \\
\hline Macrosomia & $0.99(0.81-1.21)$ & 0.903 & 1.19 (1.02-1.39) & 0.024 & $0.93(0.69-1.25)$ & 0.621 & $0.46(0.22-0.94)$ & 0.034 \\
\hline
\end{tabular}

$A O R$ adjusted odds ratio, Cl confidence interval, GDM gestational diabetes mellitus, ICP intrahepatic cholestasis of pregnancy, PTB preterm birth, SGA/LGA small/ large for gestational age, TC total cholesterol, TG triglycerides, $L D L-C / H D L-C$ low-density/high-density lipoprotein-cholesterol

Odds ratios were adjusted for maternal age, prepregnancy BMI, gestational weight gain, parity, maternal education background, family income and cigarette exposure. Values of PTB, SGA, LGA and macrosomia were additionally corrected for delivery mode and infant sex

GDM in the first trimester $(p=0.003, \mathrm{AOR}=1.58,95 \%$ CI: $1.17-2.15)$ and 1.55 -fold increased risk in the second trimester $(p=0.001, \mathrm{AOR}=1.55,95 \% \mathrm{CI}: 1.19-2.02)$. Moreover, high TG at the second trimester were significantly associated with the morbidity of ICP $(p=0.006$, $\mathrm{AOR}=1.50,95 \% \mathrm{CI}: 1.12-2.00)$. Pregnant women with higher LDL-C during the second trimester were at reduced risk of $\operatorname{GDM}(p=0.010, \mathrm{AOR}=0.48,95 \% \mathrm{CI}$ : $0.28-0.84)$.

\section{Discussion}

In this population-based study, we comprehensively displayed maternal fasting lipid profile by trimester in Chinese reproductive-age women and explored the associations between maternal lipid concentrations and pregnancy complications and perinatal outcomes. The present study confirmed maternal TG concentrations in pregnancy were positively associated with the risk of GDM, preeclampsia, LGA and macrosomia among Chinese population, which was in line with previous research performed in other races/ethnicities [14-20, 24, 31]. Furthermore, we found maternal high HDL-C concentrations were significantly associated with a decreased risk for GDM and macrosomia, and an increased risk for SGA. At the same time, hypertriglyceridemia during pregnancy was a significant predictor of ICP. These were the main novel discoveries in our study, suggesting complex metabolic disorders of maternal lipids were potentially important factors that involved in the pathological processes of adverse pregnancy outcomes.

One striking finding from this study was that maternal HDL-C concentrations were negatively associated with the risk for GDM and macrosomia, and positively associated with the risk for SGA. These associations indicated maternal HDL-C concentrations beyond a certain range was an underlying predictor of GDM, macrosomia or SGA. Very few relevant reports could be found in the past [34, 47, 48]. Among them, Wang et al. [47] believed insulin resistance, relative lack of insulin secretion and oxidative stress were the main contributors of maternal hypertriglyceridemia and low HDL-C concentrations, especially in GDM pregnancies.

Another interesting finding was that maternal high TG concentrations during the second and third trimesters were associated with an increased risk of ICP. This phenomenon has rarely been reported in the prior studies as far as we know. Evidence from a recent prospective study suggested that ICP was related with higher TG concentrations and lower HDL-C concentrations under fasting conditions [49]. Currently, theoretical explanations provided by Fiorucci et al. [50] and Sharma et al. [51] implied reduction in the activity of

Table 4 Associations between maternal HDL-C and GDM, macrosomia and SGA

\begin{tabular}{|c|c|c|c|c|c|c|}
\hline \multirow[b]{2}{*}{ Trimester } & \multicolumn{2}{|l|}{ GDM } & \multicolumn{2}{|l|}{ Macrosomia } & \multicolumn{2}{|l|}{ SGA } \\
\hline & AOR $(95 \% \mathrm{Cl})$ & $p$ value & AOR $(95 \% \mathrm{Cl})$ & $p$ value & AOR $(95 \% \mathrm{Cl})$ & $p$ value \\
\hline First & $0.15(0.05-0.42)$ & 0.000 & $0.51(0.19-1.36)$ & 0.178 & $1.31(0.32-5.38)$ & 0.709 \\
\hline Second & $0.10(0.03-0.31)$ & 0.000 & $0.25(0.09-0.73)$ & 0.011 & $1.88(0.47-7.59)$ & 0.377 \\
\hline Third & $0.27(0.13-0.58)$ & 0.001 & $0.46(0.22-0.94)$ & 0.034 & $3.15(1.15-8.65)$ & 0.026 \\
\hline
\end{tabular}

$A O R$ adjusted odds ratio, $C l$ confidence interval, GDM gestational diabetes mellitus, SGA small for gestational age, $H D L-C$ high-density lipoprotein-cholesterol Odds ratios were adjusted for maternal age, prepregnancy BMl, gestational weight gain, parity, maternal education background, family income and cigarette exposure. Values of macrosomia and SGA were additionally corrected for delivery mode and infant sex 
Table 5 Optimal cut-off points of maternal third-trimester lipids for predicting pregnancy complications and perinatal outcomes

\begin{tabular}{|c|c|c|c|c|c|}
\hline Outcomes & AUC (95 \% Cl) & Sensitivity (\%) & Specificity (\%) & Youden index & Cut-off point $(\mathrm{mmol} / \mathrm{L})$ \\
\hline \multicolumn{6}{|l|}{ TG } \\
\hline GDM & $0.633(0.566-0.701)$ & 47.9 & 74.2 & 0.221 & 3.871 \\
\hline Preeclampsia & $0.736(0.595-0.876)$ & 85.7 & 64.8 & 0.505 & 3.528 \\
\hline $\mathrm{ICP}$ & $0.607(0.528-0.685)$ & 66.1 & 55.9 & 0.220 & 3.177 \\
\hline LGA & $0.571(0.529-0.613)$ & 44.7 & 67.4 & 0.121 & 3.534 \\
\hline SGA & $0.658(0.540-0.775)$ & 63.6 & 68.9 & 0.325 & 2.530 \\
\hline \multicolumn{6}{|l|}{$\mathrm{HDL}-\mathrm{C}$} \\
\hline GDM & $0.617(0.548-0.686)$ & 57.7 & 61.2 & 0.189 & 1.712 \\
\hline Macrosomia & $0.592(0.527-0.657)$ & 66.7 & 49.8 & 0.165 & 1.817 \\
\hline SGA & $0.606(0.457-0.756)$ & 40.9 & 88.7 & 0.296 & 2.238 \\
\hline \multicolumn{6}{|l|}{ LDL-C } \\
\hline GDM & $0.649(0.582-0.715)$ & 54.9 & 71.7 & 0.266 & 2.415 \\
\hline
\end{tabular}

AUC area under curve, $C l$ confidence interval, GDM gestational diabetes mellitus, ICP intrahepatic cholestasis of pregnancy, SGA/LGA small/large for gestational age, TG triglycerides, HDL-C/LDL-C high-density/low-density lipoprotein-cholesterol

bile acid receptors FXR and TGR5 may be responsible for the above conclusion, however, the potential mechanisms still deserve further exploration.

Our study advanced the understandings of previous controversies and helped to elucidate these issues. One dispute was concerned with LDL-C concentrations in GDM pregnant women. In past studies, certain researchers observed a tendency of lower LDL-C concentrations in expectant mothers with GDM and some of them even discovered atherogenic alterations in LDL particles (increased small size, dense LDL particles and raised LDL susceptibility to oxidation) $[16,35,38]$. But there were contradictory reports stating no significant difference of LDL-C concentrations between diabetic and nondiabetic pregnancies [34,37]. For this argument, our results supported an inverse association between LDL-C concentrations and the prevalence odds of GDM, especially during the second and third trimesters. In our opinion, the conflicting consequences implied maternal lipid concentrations were influenced by complicated factors (e.g. condition of glycemic control, trimester of pregnancy and race/ethnicity).

There exists another debate focused on the correlations between maternal TG concentrations and neonatal body measurements. It has been demonstrated in some publications that maternal TG concentrations were positively correlated with neonatal birth weight or fat mass in pregnancies complicated by GDM or diabetes [28-30, 33, 39]; whereas in other articles TG concentrations were positively related with fetal development in subjects with normal glucose tolerance [31, 32]. Interestingly, many scholars once hypothesized that excessive fetal growth is derived from GDM or GIGT themselves. That means the aforementioned positive correlation may be interfered by maternal glycemic conditions. Recently, more and more studies verified the hypothesis, suggesting GDM was an independent risk factor for macrosomia [52, 53]. Consequently, our results emphasized maternal TG concentrations were independently and positively correlated with neonatal birth weight, in accordance with the viewpoints of Amsterdam Born Children and their Development cohort study [24, 54].

Compared with the existing findings, there was an inconsistent outcome in our study. In contrast with previous studies [24, 25], no significant associations were observed between maternal TG concentrations and the occurrence of PTB according to our results, whatever the gestational stage was. From our perspective, the conflicting outcomes may be explained by racial/ethnical differences, which means fetuses in different ethnic groups are disproportionally affected by maternal lipid metabolism. Future parallel studies are required to explore the underlying mechanisms of this discrepancy.

Our study has several attractive strengths. It provided some prospective evidence suggesting that dislipidemia during pregnancy places Chinese expectant mothers and their offspring at greater risk of adverse outcomes. Although lipid metabolism in pregnancy has been explored, there are controversies regarding its associations with pregnancy complications and fetal development. To our knowledge, few studies have investigated the relationship in Asian countries, particularly in China. Compared with the study conducted by Wang et al. [47] which assessed the associations between TG/HDL-C ratios and the risk of GDM and LGA, we separately identified the contributions of TG and HDL-C to pregnancy outcomes and expanded insights into effects of different lipid components on mothers and neonates. Indeed, a majority of 
existing trials focused on the concentrations of TG, TC, LDL-C and their influences on maternal and perinatal outcomes, however, less attention had been paid to HDLC. Our study not only described the variation trend of HDL-C concentrations by trimester, but also evaluated its role in developing GDM, macrosomia and SGA. Moreover, we proposed the optimal cut-off points of maternal third-trimester lipids in predicting adverse pregnancy outcomes, which was a new contribution has rarely appeared in previous research.

However, the present study still has some limitations. First of all, our sample lacked population diversity. Given that China is a country characterized by huge population and imbalanced regional economic development, a multicenter study with larger sample size can be more representative. Secondly, we selected Neonatal Birth Weight for Gestational Age and Percentile in 15 Cities of China as the criteria for LGA and SGA infants in our analysis. Defect of this criteria is its applicability worths further discussion with the increasing Chinese neonatal birth weights. Thirdly, some collected data of maternal characteristics (e.g. prepregnancy weight and GWG) were selfreported and possibly be subject to recall bias. Finally, we cannot rule out the possibility of residual confounding. For example, excessive dietary nutrition intake and inadequate physical activity during pregnancy may be important contributors of dyslipidemia, but we failed to include sufficient information on dietary nutrition intake and physical activity. Thus, additional studies are needed to clarify the relationship between maternal lifestyle and dyslipidemia and pregnancy outcomes.

\section{Conclusions}

Our results suggested that maternal high TG concentrations in late pregnancy were independently and significantly associated with an increased risk of GDM, preeclampsia, ICP, LGA, macrosomia and a decreased risk of SGA among Chinese population. Additionally, relative low HDL-C during pregnancy was significantly related with the occurence of GDM and macrosomia; whereas relative high HDL-C was a protective factor for both of them. Our findings highlighted the importance of maternal lipid metabolism in preventing pregnancy complications and adverse birth outcomes.

\footnotetext{
Abbreviations

AGA: Appropriate for gestational age; AOR: Adjusted odds ratio; AUC: Area under curve; BMI: Body mass index; CVD: Cardiovascular diseases;

DOHaD: Developmental origins of Health and Disease; GDM: Gestational diabetes mellitus; GIGT: Gestational impaired glucose tolerance; GWG: Gestational weight gain; HDL-C: High-density lipoprotein-cholesterol; ICP: Intrahepatic cholestasis of pregnancy; IQR: Interquartile range; LDL-C: Low-density lipoprotein-cholesterol; LGA: Large for gestational age; NEFA: Non-esterified fatty acids; OGTT: Oral glucose tolerance test; PTB: Preterm birth; SD: Standard deviation; SGA: Small for gestational age; TC: Total cholesterol; TG: Triglycerides.
}

\section{Competing interests}

The authors declare that they have no competing interests.

\section{Authors' contributions}

WYJ participated in study design, data acquisition, statistical analysis, literature search and drafted the manuscript. SLL participated in study design, data acquisition and literature search. RLH, XYC, TH, YJ, LT and ZWZ participated in the design of the study and the collection of the data. ZYZ conceived of the study, and participated in its design and funds collection. All the authors read and approved the final manuscript.

\section{Acknowledgements}

This study was supported by the " $11^{\text {th }}$ Five-Year Plan" and the " $12^{\text {th }}$ Five-Year Plan" from the National Science and Technology Issues Research, China (2009BAl80B03, 2012BAI02B03), the Innovation Program for Early Screening and Intervention of Birth Defects, Zhejiang Province (2010R50045), and the National Key Scientific Research Projects of China (973 Program)

(2012CB944900). We are grateful for the assistance from Department of Obstetrics, Women's Hospital, Zhejiang University School of Medicine in data collection and the participation of all the subjects in our study. We also thank Professor Colin Binns and Professor Andy Lee from School of Public Health,

Curtin University, Australia for their guidance in statistical analysis.

\section{Author details}

'Department of Children's Health Care, Children's Hospital, Zhejiang University School of Medicine, Hangzhou 310003, China. '2 Department of Epidemiology and Biostatistics, School of Public Health, Curtin University, Perth WA6845, Australia.

Received: 11 May 2015 Accepted: 17 March 2016

Published online: 21 March 2016

\section{References}

1. Kaaja RJ, Greer IA. Manifestations of chronic disease during pregnancy. JAMA. 2005;294(21):2751-7.

2. Lekva T, Bollerslev J, Norwitz ER, Aukrust P, Henriksen T, Ueland T. Aortic Stiffness and Cardiovascular Risk in Women with Previous Gestational Diabetes Mellitus. PLoS One. 2015;10(8), e0136892.

3. Zoet GA, Koster MP, Velthuis BK, de Groot CJ, Maas AH, Fauser BC, et al. Determinants of future cardiovascular health in women with a history of preeclampsia. Maturitas. 2015;82(2):153-61.

4. Charlton F, Tooher J, Rye KA, Hennessy A. Cardiovascular risk, lipids and pregnancy: preeclampsia and the risk of later life cardiovascular disease. Heart Lung Circ. 2014;23(3):203-12.

5. Yang JJ, Lee SA, Choi JY, Song M, Han S, Yoon HS, et al. Subsequent risk of metabolic syndrome in women with a history of preeclampsia: data from the Health Examinees Study. J Epidemiol. 2015;25(4):281-8.

6. Gillman MW. Developmental origins of health and disease. N Engl J Med. 2005;353(17):1848-50.

7. Prentice AM, Moore SE. Early programming of adult diseases in resource poor countries. Arch Dis Child. 2005;90(4):429-32.

8. Bartels Ä, Egan N, Broadhurst DI, Khashan AS, Joyce C, Stapleton M, et al. Maternal serum cholesterol levels are elevated from the 1st trimester of pregnancy: A cross-sectional study. J Obstet Gynaecol. 2012;32(8):747-52.

9. Brizzi P, Tonolo G, Esposito F, Puddu L, Dessole S, Maioli M, Milia S. Lipoprotein metabolism during normal pregnancy. Am J Obstet Gynecol. 1999;181(2):430-4.

10. Piechota W, Staszewski A. Reference ranges of lipids and apolipoproteins in pregnancy. Eur J Obstet Gynecol Reprod Biol. 1992;45(1):27-35.

11. Lippi G, Albiero A, Montagnana M, Salvagno GL, Scevarolli S, Franchi M, Guidi GC. Lipid and lipoprotein profile in physiological pregnancy. Clin Lab. 2007;53(3-4):173-7.

12. Husain $F$, Latif $S$, Uddin M, Nessa A. Lipid profile changes in second trimester of pregnancy. Mymensingh Med J. 2008;17(1):17-21.

13. Ghio A, Bertolotto A, Resi V, Volpe L, Di Cianni G. Triglyceride metabolism in pregnancy. Adv Clin Chem. 2011;55:133-53.

14. Hollingsworth DR, Grundy SM. Pregnancy-associated hypertriglyceridemia in normal and diabetic women. Differences in insulin-dependent, non-insulindependent, and gestational diabetes. Diabetes. 1982;31(12):1092-7. 
15. Koukkou E, Watts GF, Lowy C. Serum lipid, lipoprotein and apolipoprotein changes in gestational diabetes mellitus: a cross-sectional and prospective study. J Clin Pathol. 1996;49(8):634-7.

16. Sánchez-Vera I, Bonet B, Viana M, Quintanar A, Martín MD, Blanco P, et al. Changes in plasma lipids and increased low-density lipoprotein susceptibility to oxidation in pregnancies complicated by gestational diabetes: consequences of obesity. Metabolism. 2007;56(11):1527-33.

17. Wiznitzer A, Mayer A, Novack V, Sheiner E, Gilutz H, Malhotra A, Novack L. Association of lipid levels during gestation with preeclampsia and gestational diabetes mellitus: a population-based study. Am J Obstet Gynecol. 2009;201(5):482.e1-8.

18. Hubel CA, McLaughlin MK, Evans RW, Hauth BA, Sims CJ, Roberts JM. Fasting serum triglycerides, free fatty acids, and malondialdehyde are increased in preeclampsia, are positively correlated, and decrease within 48 hours post partum. Am J Obstet Gynecol. 1996;174(3):975-82.

19. Baker AM, Klein RL, Moss KL, Haeri S, Boggess K. Maternal serum dyslipidemia occurs early in pregnancy in women with mild but not severe preeclampsia. Am J Obstet Gynecol. 2009;201(3):293.e1-4.

20. Spracklen CN, Smith CJ, Saftlas AF, Robinson JG, Ryckman KK. Maternal hyperlipidemia and the risk of preeclampsia: a meta-analysis. Am J Epidemiol. 2014:180(4):346-58.

21. Gratacós E, Casals E, Gómez O, Llurba E, Mercader I, Cararach V, Cabero L. Increased susceptibility to low density lipoprotein oxidation in women with a history of pre-eclampsia. BJOG. 2003;110(4):400-4

22. Sanchez SE, Williams MA, Muy-Rivera M, Qiu C, Vadachkoria S, Bazul V. A case-control study of oxidized low density lipoproteins and preeclampsia risk. Gynecol Endocrinol. 2005;21(4):193-9.

23. Qiu C, Phung TT, Vadachkoria S, Muy-Rivera M, Sanchez SE, Williams MA Oxidized low-density lipoprotein (oxidized LDL) and the risk of preeclampsia. Physiol Res. 2006;55(5):491-500

24. Vrijkotte TG, Krukziener N, Hutten BA, Vollebregt KC, van Eijsden M, Twickler MB. Maternal lipid profile during early pregnancy and pregnancy complications and outcomes: the ABCD study. J Clin Endocrinol Metab. 2012:97(11):3917-25.

25. Catov JM, Bodnar LM, Kip KE, Hubel C, Ness RB, Harger G, Roberts JM. Early pregnancy lipid concentrations and spontaneous preterm birth. Am J Obstet Gynecol. 2007;197(6):610.e1-7.

26. Herrera E. Lipid metabolism in pregnancy and its consequences in the fetus and newborn. Endocrine. 2002;19(1):43-55.

27. Herrera E, Ortega-Senovilla H. Lipid metabolism during pregnancy and its implications for fetal growth. Curr Pharm Biotechnol. 2014;15(1):24-31.

28. Herrera E, Ortega-Senovilla H. Disturbances in lipid metabolism in diabetic pregnancy - Are these the cause of the problem? Best Pract Res Clin Endocrinol Metab. 2010;24(4):515-25.

29. Schaefer-Graf UM, Graf K, Kulbacka I, Kjos SL, Dudenhausen J, Vetter K, Herrera E. Maternal lipids as strong determinants of fetal environment and growth in pregnancies with gestational diabetes mellitus. Diabetes Care. 2008; 31(9):1858-63.

30. Ortega-Senovilla H, Schaefer-Graf U, Meitzner K, Abou-Dakn M, Herrera E. Decreased concentrations of the lipoprotein lipase inhibitor angiopoietinlike protein 4 and increased serum triacylglycerol are associated with increased neonatal fat mass in pregnant women with gestational diabetes mellitus. J Clin Endocrinol Metab. 2013:98(8):3430-7.

31. Kitajima M, Oka S, Yasuhi I, Fukuda M, Rii Y, Ishimaru T. Maternal serum triglyceride at 24-32 weeks' gestation and newborn weight in nondiabetic women with positive diabetic screens. Obstet Gynecol. 2001;97(5 Pt 1):776-80.

32. Di Cianni G, Miccoli R, Volpe L, Lencioni C, Ghio A, Giovannitti MG, et al. Maternal triglyceride levels and newborn weight in pregnant women with normal glucose tolerance. Diabet Med. 2005;22(1):21-5.

33. Göbl CS, Handisurya A, Klein K, Bozkurt L, Luger A, Bancher-Todesca D, Kautzky-Willer A. Changes in serum lipid levels during pregnancy in type 1 and type 2 diabetic subjects. Diabetes Care. 2010;33(9):2071-3.

34. Ryckman KK, Spracklen CN, Smith CJ, Robinson JG, Saftlas AF. Maternal lipid levels during pregnancy and gestational diabetes: a systematic review and meta-analysis. BJOG. 2015;122(5):643-51.

35. Qiu C, Rudra C, Austin MA, Williams MA. Association of gestational diabetes mellitus and low-density lipoprotein (LDL) particle size. Physiol Res. 2007:56(5):571-8

36. Savona-Ventura C, Vassallo J, Craus J, Anastasiou E, Jotic A, Lalic NM, et al. Biological and biochemical characteristics of a Mediterranean population with Gestational Diabetes Mellitus. J Perinat Med. 2015. doi: 10.1515/jpm-2015-0027.

37. Retnakaran R, Qi Y, Connelly PW, Sermer M, Hanley AJ, Zinman B. The graded relationship between glucose tolerance status in pregnancy and postpartum levels of low-density lipoprotein cholesterol and apolipoprotein B in young women: implications for future cardiovascular risk. J Clin Endocrinol Metab. 2010;95(9):4345-53.

38. Rizzo M, Berneis K, Altinova AE, Toruner FB, Akturk M, Ayvaz G, et al. Atherogenic lipoprotein phenotype and LDL size and subclasses in women with gestational diabetes. Diabet Med. 2008;25(12):1406-11.

39. Nolan CJ, Riley SF, Sheedy MT, Walstab JE, Beischer NA. Maternal serum triglyceride, glucose tolerance and neonatal birth weight ratio in pregnancy. Diabetes Care. 1995;18(12):1550-6.

40. World Health Organization. Obesity: preventing and managing the global epidemic. Report of a WHO Consultation. World Health Organ Tech Rep Ser. 2000; 894:i-xii,1-253.

41. Institute of Medicine (US) and National Research Council (US) Committee to Reexamine IOM Pregnancy Weight Guidelines, Rasmussen KM, Yaktine AL, et al. Weight Gain During Pregnancy: Reexamining the Guidelines. Washington (DC): National Academies Press (US); 2009.

42. International Association of Diabetes and Pregnancy Study Groups Consensus Panel, Metzger BE, Gabbe SG, et al. International association of diabetes and pregnancy study groups recommendations on the diagnosis and classification of hyperglycemia in pregnancy. Diabetes Care. 2010; 33(3):676-82.

43. Wei Y, Yang $\mathrm{H}$, Zhu W, Yang H, Li H, Yan J, Zhang C. International Association of Diabetes and Pregnancy Study Group criteria is suitable for gestational diabetes mellitus diagnosis: further evidence from China. Chin Med J (Engl). 2014;127(20):3553-6.

44. Eiland E, Nzerue C, Faulkner M. Preeclampsia 2012. J Pregnancy. 2012; 2012:586578.

45. Williamson C, Geenes V. Intrahepatic cholestasis of pregnancy. Obstet Gynecol. 2014;124(1):120-33.

46. Scientific research collaborative group of neonatal development in 15 cities of China. Neonatal birth weight for gestational age and percentile in 15 cities of China. Zhonghua Er Ke Za Zhi (Chinese). 1989;27:316

47. Wang D, Xu S, Chen H, Zhong L, Wang Z. The associations between triglyceride to high-density lipoprotein cholesterol ratios and the risks of gestational diabetes mellitus and large-for-gestational-age infant. Clin Endocrinol (Oxf). 2015;83(4):490-7.

48. Li G, Kong L, Zhang L, Fan L, Su Y, Rose JC, Zhang W. Early pregnancy maternal lipid profiles and the risk of gestational diabetes mellitus stratified for body mass index. Reprod Sci. 2015;22(6):712-7.

49. Martineau MG, Raker C, Dixon PH, Chambers J, Machirori M, King NM, et al. The metabolic profile of intrahepatic cholestasis of pregnancy is associated with impaired glucose tolerance, dyslipidemia, and increased fetal growth. Diabetes Care. 2015;38(2):243-8.

50. Fiorucci S, Mencarelli A, Palladino G, Cipriani S. Bile-acid-activated receptors: targeting TGR5 and farnesoid-X-receptor in lipid and glucose disorders. Trends Pharmacol Sci. 2009:30(11):570-80.

51. Sharma R, Long A, Gilmer JF. Advances in bile acid medicinal chemistry. Curr Med Chem. 2011;18(26):4029-52.

52. Wahabi HA, Fayed AA, Alzeidan RA, Mandil AA. The independent effects of maternal obesity and gestational diabetes on the pregnancy outcomes. BMC Endocr Disord. 2014;14:47.

53. He XJ, Qin FY, Hu CL, Zhu M, Tian CQ, Li L. Is gestational diabetes mellitus an independent risk factor for macrosomia: a meta-analysis? Arch Gynecol Obstet. 2015;291(4):729-35.

54. Vrijkotte TG, Algera SJ, Brouwer IA, van Eijsden M, Twickler MB. Maternal triglyceride levels during early pregnancy are associated with birth weight and postnatal growth. J Pediatr. 2011:159(5):736-42. 